Network Working Group

Request for Comments: 1404

B. Stockman

NORDUnet/SUNET

January 1993

A Model for Common Operational Statistics

Status of the Memo

This memo provides information for the Internet community. It does not specify an Internet standard. Distribution of this memo is unlimited.

Abstract

This memo describes a model for operational statistics in the Internet. It gives recommendations for metrics, measurements, polling periods, storage formats and presentation formats.

Acknowledgements

The author would like to thank the members of the Operational Statistics Working Group of the IETF whose efforts made this memo possible.

Table of Contents

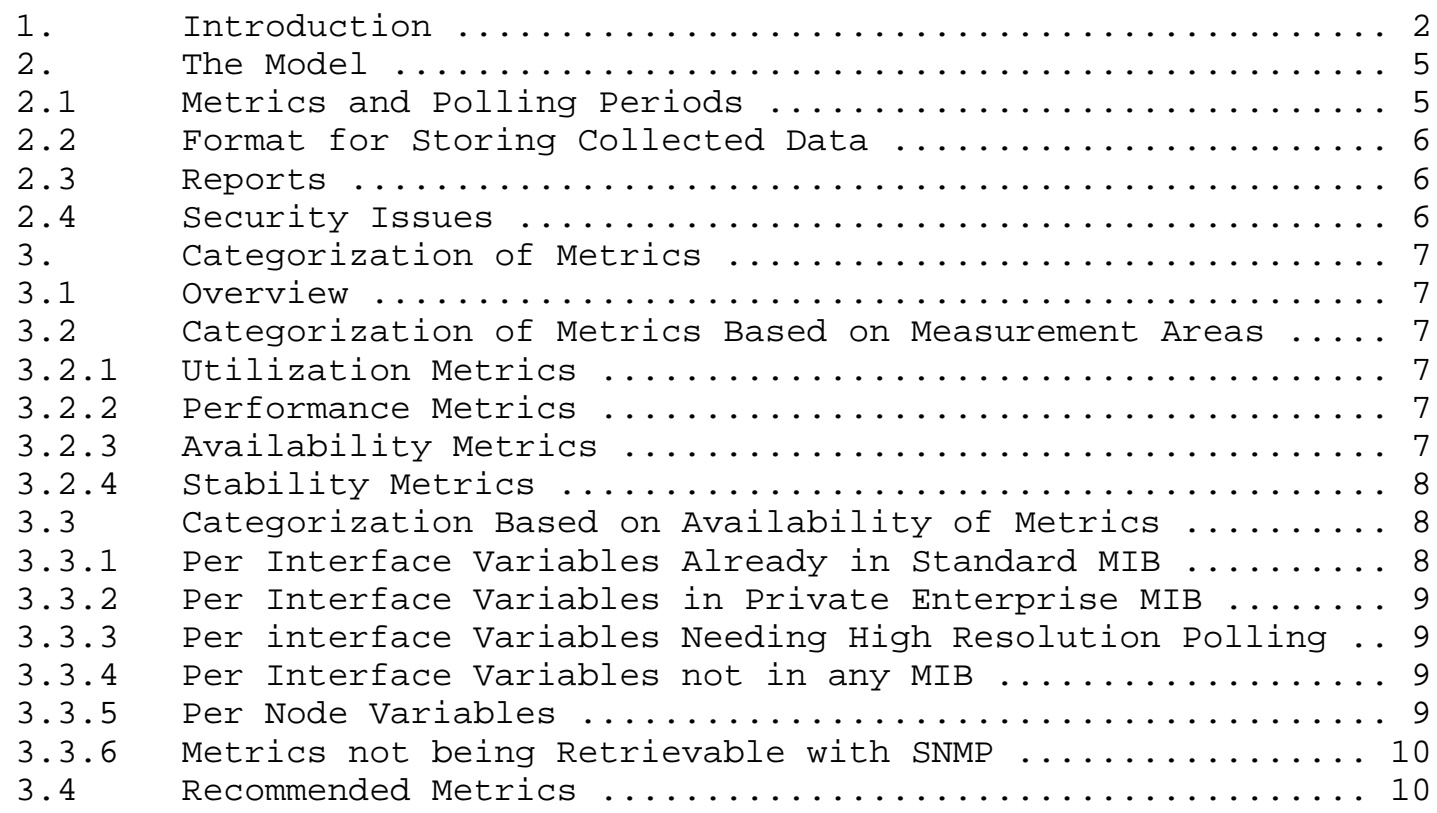




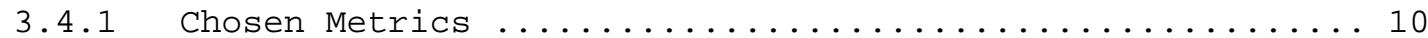

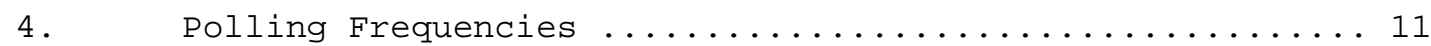

4.1 Variables Needing High Resolution Polling ............ 11

4.2 Variables not Needing High Resolution Polling ......... 11

5. Pre-Processing of Raw Statistical Data ............ 12

5.1 Optimizing and Concentrating Data to Resources ....... 12

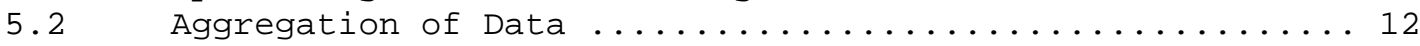

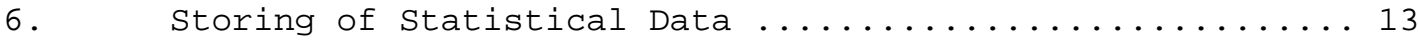

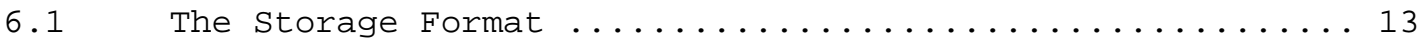

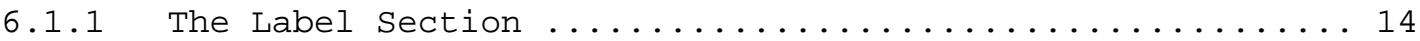

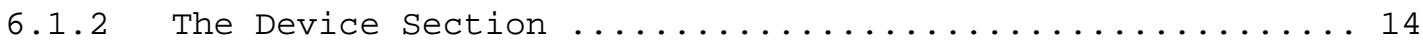

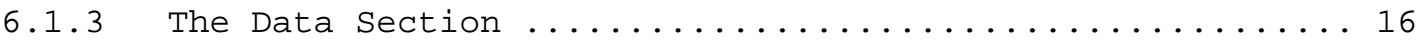

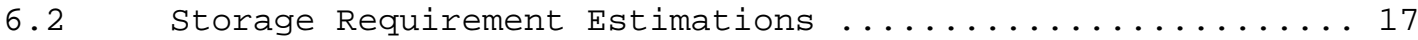

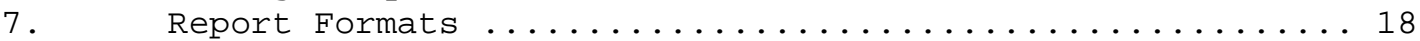

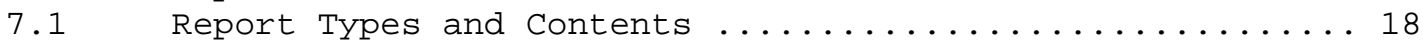

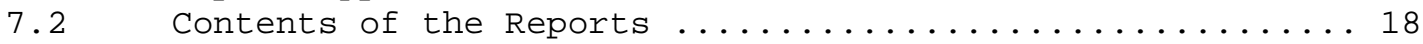

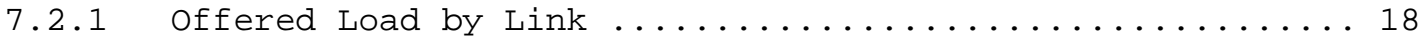

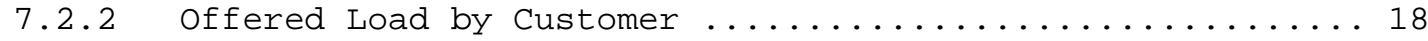

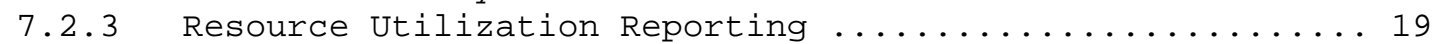

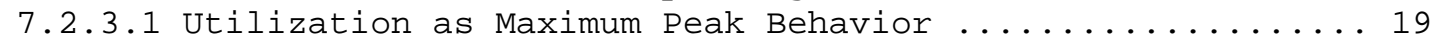

7.2.3.2 Utilization as Frequency Distribution of Peaks ....... 19

8. Considerations for Future Development ............. 20

8.1 A Client/Server Based Statistical Exchange System ...... 20

8.2 Inclusion of Variables not in the Internet Standard MIB . 20

8.3 Detailed Resource Utilization Statistics ........... 20

Appendix A Some formulas for statistical aggregation ........ 21

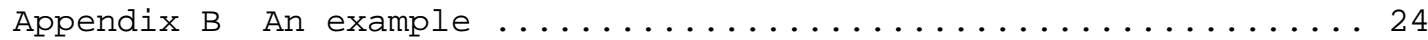

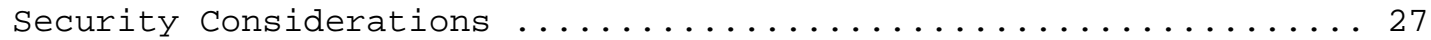

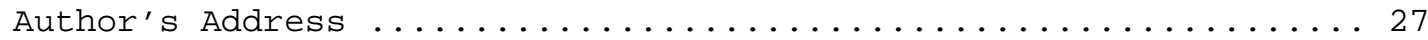

1. Introduction

Today it is not uncommon for many network administrations to collect and archive network management metrics that indicate network utilization, growth, and outages. The primary goal is to facilitate near-term problem isolation and longer-term network planning within the organization. There is also the larger goal of cooperative problem isolation and network planning between network administrations. This larger goal is likely to become increasingly important as the Internet continues to grow.

There exist a variety of network management tools for the collection and presentation of network management metrics. However, different kinds of measurement and presentation techniques makes it difficult to compare data between networks. Plus, there is not common agreement on what metrics should be regularly collected or how they should be displayed. 
There needs to be an agreed-upon model for

1) A minimal set of common network management metrics to satisfy the goals stated above.

2) Tools for collecting these metrics.

3) A common storage format to facilitate the usage of these data by common presentation tools.

4) Common presentation formats.

Under this operational Statistics model, collection tools will collect and store data in a given format to be retrieved later by presentation tools displaying the data in a predefined way. (See figure below.) 
The Operational Statistics Model

(Collection of common metrics, by commonly available tools, stored in a common format, displayed in common formats by commonly available presentation tools.)

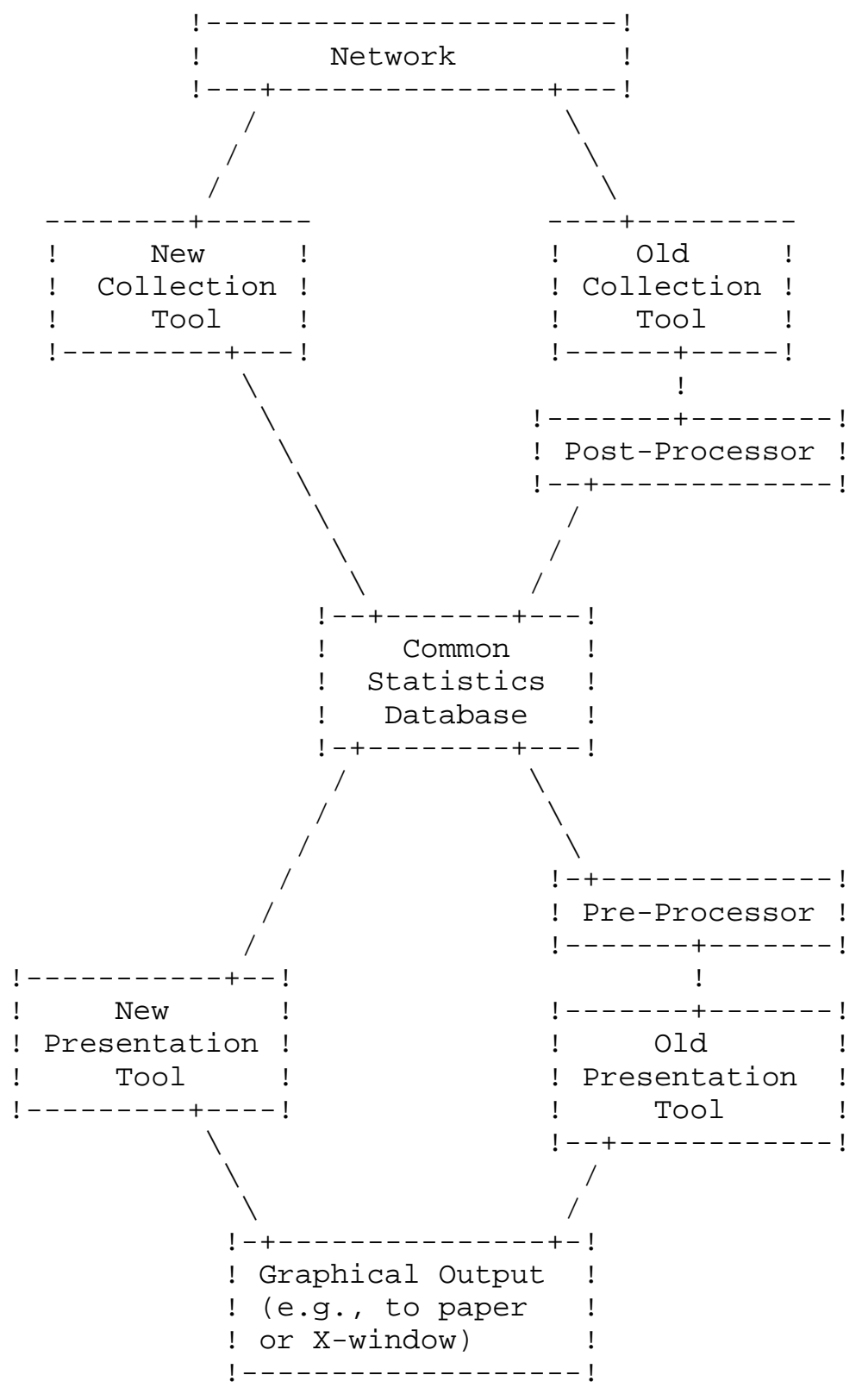

Stockman

[Page 4] 
This memo gives an overview of this model for common operational statistics. The model defines the gathering, storing and presentation of network operational statistics and classifies the types of information that should be available at each network operation center conforming to this model.

The model defines a minimal set of metrics, how these metrics should gathered and stored. Finally the model gives recommendations on the content and the layout of statistical reports making it possible to easily compare networks statistics between NoCs.

The primary purpose of this model is to define ways and methods on how NOCs could most effectively share their operational statistics. One intention with this model is to specify a baseline capability that NOCs conforming to the this model may support with a minimal development effort and a minimal ongoing effort.

2. The Model

The model defines three areas of interest on which all underlying concepts are based.

1. The definition of a minimal set of metrics to be gathered

2. The definition of a format for storing collected statistical data.

3. The definition of methods and formats for generating reports.

The model indicates that old tools used today could be retrofitted into the new paradigm. This could be done by providing conversionfilters between the old and the new environment tools. In this sense this model intends to advocate the development of public domain software for use by participating NoCs.

One basic idea with the model is that statistical data stored at one place could be retrieved and displayed at some other place.

2.1 Metrics and Polling Periods

The intention here is to define a minimal set of metrics that easily could be gathered using standard SNMP based network management tools. These metrics should hence be available as variables in the Internet Standard MIB.

If the Internet standard MIB is changed also this minimal set of metrics could be reconsidered as there are many metrics viewed as 
important but currently not being defined in the standard MIB. For some metrics being highly desirable to collect there are currently no way to get them into the Internet standard MIB as these metrics probably are not possible to retrieve using SNMP. Tools and methods in gathering such metrics should be explicitly defined if such metrics are to be considered. This is, however, outside of the scope of this memo.

\subsection{Format for Storing Collected Data}

A format for storing data is defined. The intention is to minimize redundant information by using a single header structure where all information relevant to a certain set of statistical data is stored. This header section will give information on when and where the corresponding statistical data where collected.

\subsection{Reports}

Some basic classes of reports are suggested with regards to different views of network behavior. For this reason reports on totals of octets and packets over some period in time are regarded as essential to give an overall view of the traffic flows in a network. Differentiation between application and protocols to give ideas on which type of traffic is dominant is regarded as needed. Finally reports on resource utilization are recommended..

Depending on the intention with a report the timeperiod over which it spans may vary. For capacity planning there may be a need for longer term reports while in engineering and operation there may be sufficient with reports on weekly or daily basis.

\subsection{Security Issues}

There are legal, ethical and political concerns of data sharing. People are concerned about showing data that may make one of the networks look bad.

For this reason there is a need to insure integrity, conformity and confidentiality of the shared data. To be useful, the same data must be collected from all of the involved sites and it must be collected at the same interval. To prevent vendors from getting an unfair performance information, certain data must not be made available. 


\section{Categorization of Metrics}

\subsection{Overview}

This section gives a classification of metrics with regard to scope and easiness of retrieve. A recommendation of a minimal set of metrics is given. The section also gives some hints on metrics to be considered for future inclusion when available in the network management environment. Finally some thoughts on storage requirements are presented.

\subsection{Categorization of Metrics Based on Measurement Areas}

The metrics used in evaluating network traffic could be classified into (at least) four major categories:

- Utilization metrics

- Performance metrics

- Availability metrics

- Stability metrics

\subsubsection{Utilization Metrics}

These category describes different aspects of the total traffic being forwarded through the network. Possible metrics are:

- Total input and output packets and octets.

- Various peak metrics.

- Per protocol and per application metrics.

\subsubsection{Performance Metrics}

These metrics describes the quality of service such as delays and congestion situations. Possible metrics are:

- RTT metrics on different protocol layers.

- Number of collisions on a bus network

- Number of ICMP Source Quench messages.

- Number of packets dropped.

- etc.

\subsubsection{Availability Metrics}

This could be considered as the long term accessibility metrics on different protocol layers. Possible metrics are: 
- Line availability as percentage uptime.

- Route availability

- Application availability

\subsubsection{Stability Metrics}

These metrics describes short term fluctuations in the network which degrades the service level. Also changes in traffic patterns could be recognized using these metrics. Possible metrics are:

- Number of fast line status transitions

- Number of fast route changes (also known as route flapping)

- Number of routes per interface in the tables

- Next hop count stability.

- Short term ICMP behaviors.

\subsection{Categorization Based on Availability of Metrics}

To be able to retrieve metrics the corresponding variables must be possible to access at every network object being part of the management domain for which statistics are being collected.

Some metrics are easily retrievable as being defined as variables in the Internet standard MIB while other metrics may be retrievable as being part of some vendor's private enterprise MIB subtree. Finally some metrics are considered as impossible to retrieve due to not being possible to include in the SNMP concept or that the actual measurement of these metrics would require extensive polling and hence download the network with management traffic.

The metrics being categorized below could each be judged as an important metric in evaluating network behaviors. This list may serve for reconsider the decisions on which metric to be regarded as reasonable and desirable to collect. If the availability of below metrics changes these decisions may change.

3.3.1 Per Interface Variables Already in Internet Standard MIB (thus easy to retrieve)

$\begin{array}{ll}\text { ifInUcastPkts } & \text { (unicast packet in) } \\ \text { ifOutUcastPkts } & \text { (unicast packet out) } \\ \text { ifInNUcastPkts } & \text { (non-unicasts packet in } \\ \text { ifOutNUcastPkts } & \text { (non-unicast packet out) } \\ \text { ifInOctets } & \text { (octets in) } \\ \text { ifOutOctets } & \text { (octets out) } \\ \text { ifOperstatus } & \text { (line status) }\end{array}$


3.3.2 Per Interface Variables in Internet Private Enterprise MIB

(thus could sometimes be possible to retrieve)

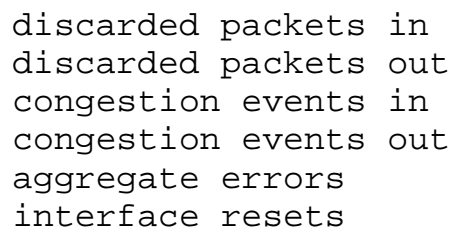

3.3.3 Per Interface Variables Needing High Resolution Polling

(which is hard due to resulting network load)

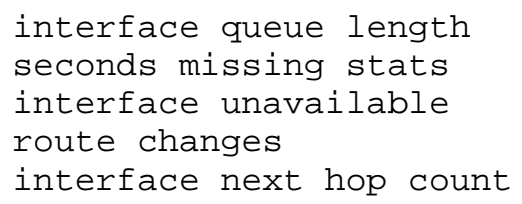




\subsubsection{Metrics not being Retrievable with SNMP}

delays (RTTs) on different protocol layers

application layer availabilities

peak behavior metrics

\section{4 Recommended Metrics}

A large amount of metrics could be regarded for gathering in the process of doing network statistics. To facilitate for this model to reach general consensus there is a need to define a minimal set of metrics that are both essential and also possible to retrieve in a majority of today network objects. As an indication of being generally retrievable the presence in the Internet standard MIB is regarded as a mandatory requirement.

\subsubsection{Chosen Metrics}

The following metrics were chosen as desirable and reasonable being part of the Internet Standard MIB:

For each interface:

$\begin{array}{ll}\text { ifInOctets } & \text { (octets in) } \\ \text { ifOutOctets } & \text { (octets out) } \\ \text { ifInUcastPkts } & \text { (unicast packets in) } \\ \text { ifOutUcastPkts } & \text { (unicast packets out) } \\ \text { ifInNUcastPkts } & \text { (non-unicast packets in) } \\ \text { ifOutNUcastPkts } & \text { (non-unicast packets out) } \\ \text { ifInDiscards } & \text { (in discards) } \\ \text { ifOutDiscards } & \text { (out discards) } \\ \text { ifoperStatus } & \text { (line status) }\end{array}$

For each node:

$\begin{array}{ll}\text { ipForwDatagrams } & \text { (IP forwards) } \\ \text { ipInDiscards } & \text { (IP in discards) } \\ \text { sysUpTime } & \text { (system uptime) }\end{array}$

All of the above metrics are available in the Internet Standard MIB. However, there also other metrics which could be recommended such as the RTT metric which probably never will be in any MIB. For such metrics other collection tools than SNMP have to be explicitly defined. The specification of such tools are outside scope of this memo. 


\section{Polling Frequencies}

The reason for the polling is to achieve statistics to serve as base for trend and capacity planning. From the operational data it shall be possible to derive engineering and management data. It shall be noted that all polling and saving values below are recommendation and not mandatory.

\subsection{Variables Needing High Resolution Polling}

To be able to detect peak behaviors it is recommended that a period of maximum 1 minute (60 seconds) is used in the gathering of traffic data. The metrics to be gathered at this frequency is:

for each interface

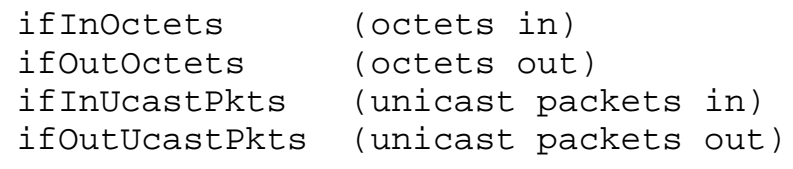

If not possible to gather data at this high polling frequency, it is recommended that an even multiple of 60 seconds is used. The initial polling frequency value will be part of the stored statistical data as described in section 4 below.

4.2 Variables not Needing High Resolution Polling

The other part of the recommended variables to be gathered, i.e.,

For each interface:

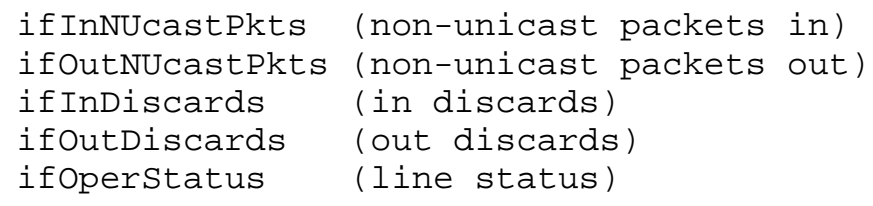

and for each node:

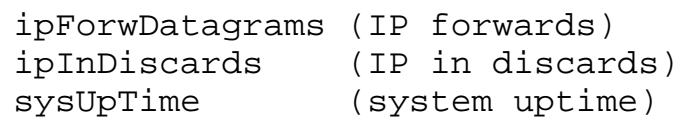

These variables could be gathered at a lower polling rate. No specific polling rate is mentioned but it is recommended that the period chosen is an even multiple of 60 seconds. 
5. Pre-Processing of Raw Statistical Data

\subsection{Optimizing and Concentrating Data to Resources}

To avoid redundant data being stored in commonly available storage there is a need for processing the raw data. For example if a link is down there is no need to continuous store a counter that is not changing. Using variables such as sysuptime and Line status there is the possibility of not continuously storing data collected from links and nodes where no traffic have been transmitted over some period of time.

Another aspect of processing is to decouple the data from the raw interface being polled. The intention should be to convert such data into the resource being of interest as for example the traffic on a given link. Changes of interface in a gateway for a given link should not be visible in the provided data.

\subsection{Aggregation of Data}

A polling period of 1 minute will create the need of aggregating stored data. Aggregation here means that over a period with logged entries, a new aggregated entry is created by taking the first and last of the previously logged entries over some aggregation period and compute a new entry.

Not to loose information on the peak values the aggregation also means that the peak value of the previous aggregation period is calculated and stored.

This gives below layout of aggregated entries

It is foreseen that over a relatively short period, polled data will be logged at the tightest polling period (1 minute). Regularly these data will be pre-processed into the actual files being provided.

Suggestions for aggregation periods:

Over a
24 hour period
aggregate to 15 minutes,
1 month period aggregate to 1 hour,
1 year period aggregate to 1 day

Aggregation is the computation of new average and maximum values for the aggregation period based on the previous aggregation period data. For each aggregation period the maximum, and average values are computed and stored. Also other aggregation period could be chosen 
when needed. The chosen aggregation period value will be stored together with the aggregated data as described below.

6. Storing of Statistical Data

This section describes a format for storing of statistical data. The goal is to facilitate for a common set of tools for the gathering, storing and analysis of statistical data. The format is defined with the intention to minimize redundant information and by this minimize required storage. If a client server based model for retrieving remote statistical data is later being developed, the specified storage format should be possible to used as the transmission protocol.

The format is built up by three different sections within the statistical storage, a label section, a device section and a data section. The label section gives the start and end times for a given data section as well as the file where the actual data is stored. The device section specifies what is being logged in the corresponding data section.

To facilitate for multiple data sections within one log-file, label sections, device sections and data sections may occur more than once. Each section type is delimited by a BEGIN-END pair. Label and device sections could either be stored directly in the data-file or as separate files where the corresponding data-file is pointed out by the data-file entry in the label section.

A data section must correspond to exactly one label section and one device section. If more label sections and device sections each data section will belong to the label section and device section immediately prepending the data section if these sections are stored within the data-file. How files are physically arranged is outside the scope of the document.

6.1 The Storage Format

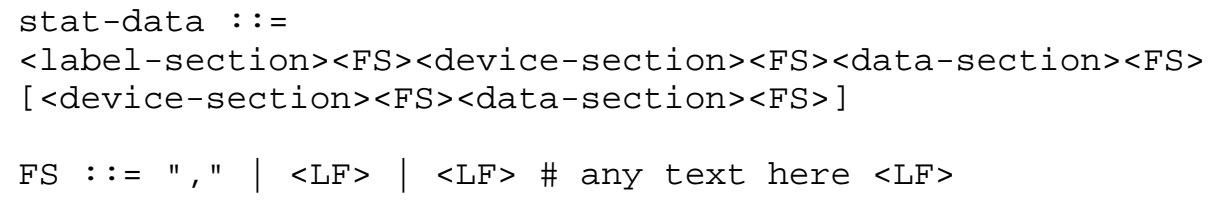


specification.

It shall here be noted that the actual physical storage of data is a local decision and can vary a lot. There can be one data-file per interface or multiple interfaces logged within the same data-file. Label and device sections may be stored in a separate file as well as within the data-file.

6.1.1 The Label Section

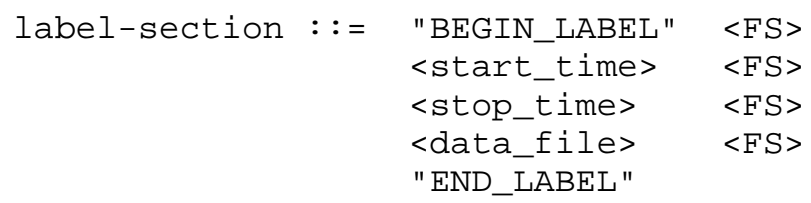

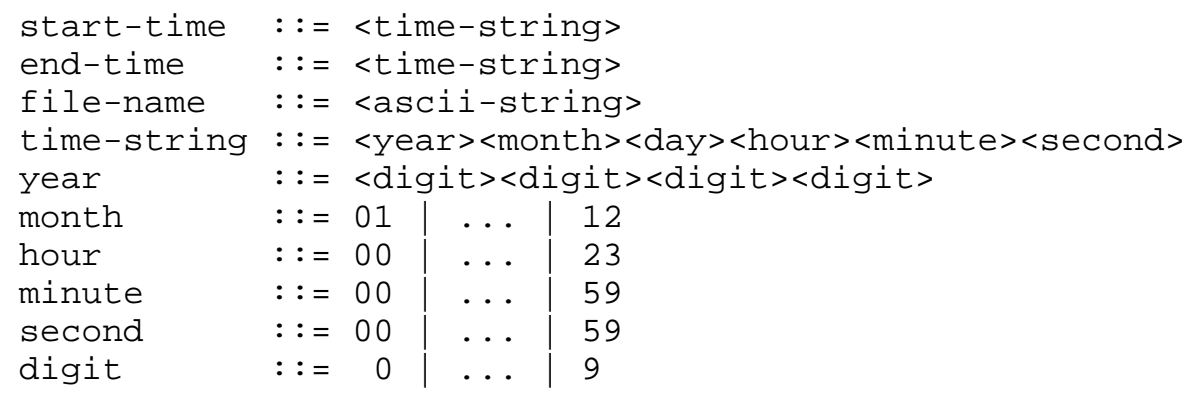

\subsubsection{The Device Section}

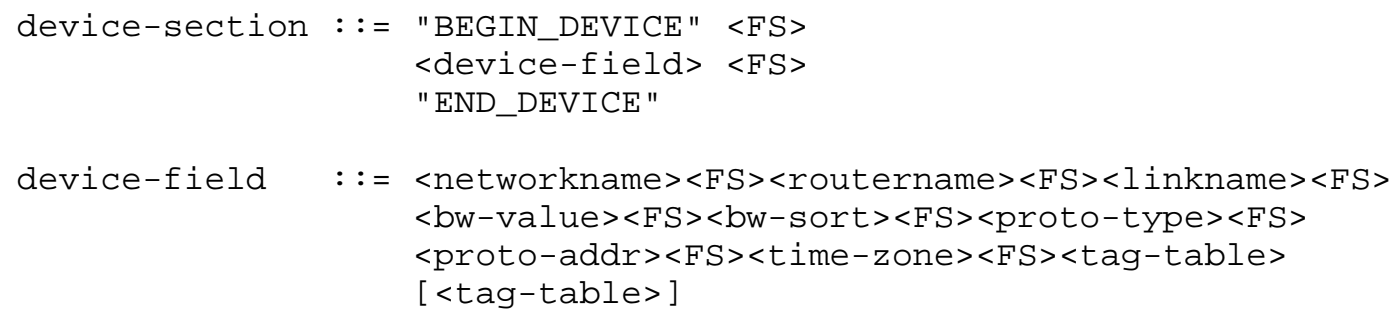




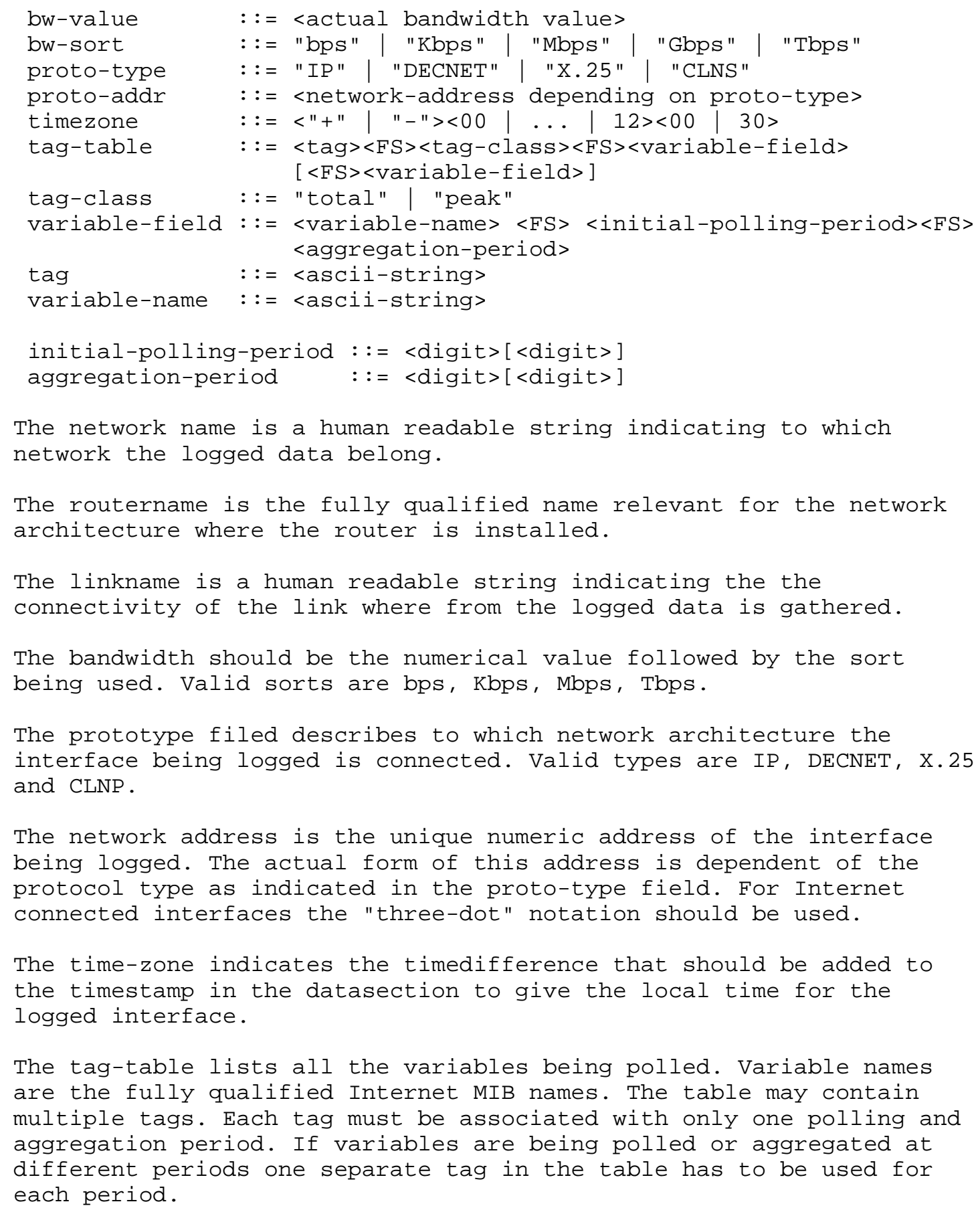

The network name is a human readable string indicating to which network the logged data belong.

The routername is the fully qualified name relevant for the network architecture where the router is installed.

The linkname is a human readable string indicating the the connectivity of the link where from the logged data is gathered.

The bandwidth should be the numerical value followed by the sort being used. Valid sorts are bps, Kbps, Mbps, Tbps.

The prototype filed describes to which network architecture the interface being logged is connected. Valid types are IP, DECNET, X.25 and CLNP.

The network address is the unique numeric address of the interface being logged. The actual form of this address is dependent of the protocol type as indicated in the proto-type field. For Internet connected interfaces the "three-dot" notation should be used.

The time-zone indicates the timedifference that should be added to the timestamp in the datasection to give the local time for the logged interface.

The tag-table lists all the variables being polled. Variable names are the fully qualified Internet MIB names. The table may contain multiple tags. Each tag must be associated with only one polling and aggregation period. If variables are being polled or aggregated at different periods one separate tag in the table has to be used for each period. 
As variables may be polled with different polling periods within the same set of logged data, there is a need to explicitly associate a polling period with each variable. After being processed the actual period covered may have changed as compared to the initial polling period and this should be noted in the aggregation period field. The initial polling period and aggregation period should be given in seconds.

As aggregation also means the computation of the max value for the previously polled data, the aggregation process have to extend the tag table to include these maximum values. This could be done in different ways. The variable field for the aggregated variables is extended to also include the peak values from the previous period. Another possibility is to create new tags for the peak values. To be able to differentiate between polled raw data, aggregated total and aggregated peak values some kind of unique naming of such entities has to be implemented.

6.1.3 The Data Section

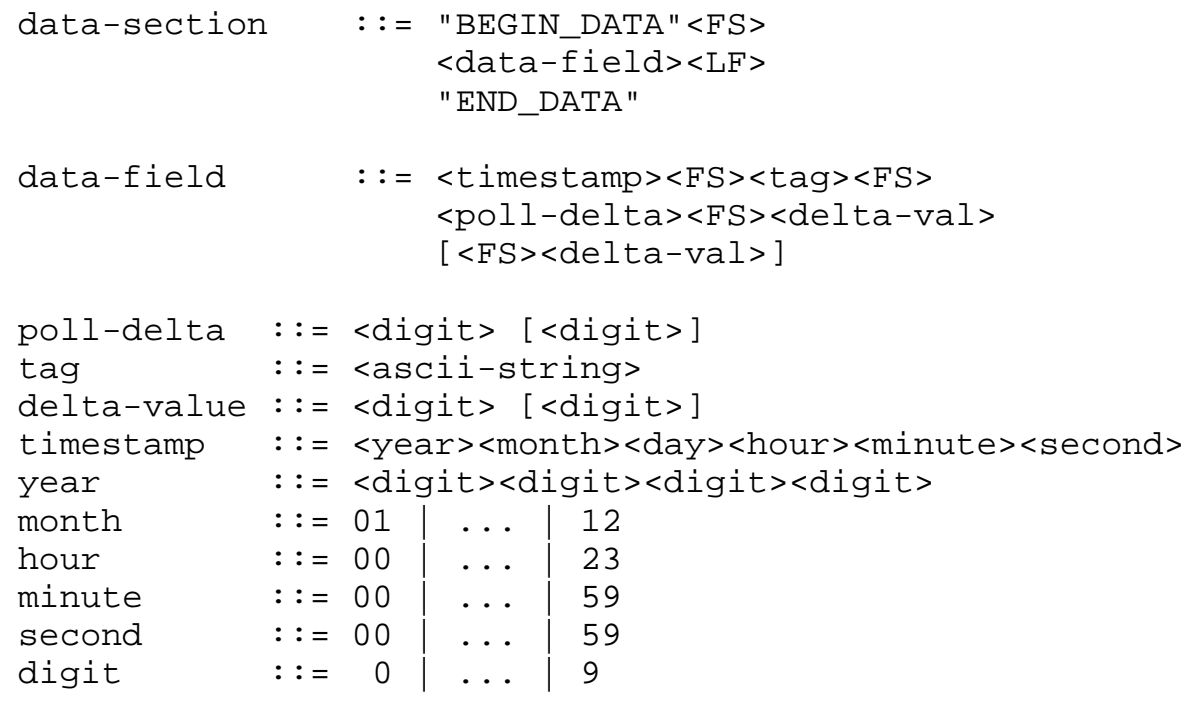

The datafield contains the polled data from a set of variables as defined by the corresponding tag field. Each data field begins with the timestamp for this poll followed by the tag defining the polled variables followed by a polling delta value giving the period of time in seconds since the previous poll. The variable values are stored as delta values for counters and as absolute values for non-counter values such as OperStatus. The timestamp is in UTC and the time-zone field in the device section is used to compute the local time for the device being logged. 


\subsection{Storage Requirement Estimations}

The header sections are not counted in this example. Assuming the the maximum polling intensity is used for all the 12 recommended variables and assuming the size in ascii of each variable is 8 bytes will give the below calculations based on one year of storing and aggregating statistical data.

Assuming that data is saved according to the below scheme

$\begin{array}{ll}1 \text { minute non-aggregated } & \text { saved } 1 \text { day. } \\ 15 \text { minute aggregation period } & \text { saved } 1 \text { week. } \\ 1 \text { hour aggregation period } & \text { saved } 1 \text { month. } \\ 1 \text { day aggregation period } & \text { saved } 1 \text { year. }\end{array}$

this will give:

Size of one entry for each aggregation period:

Aggregation periods

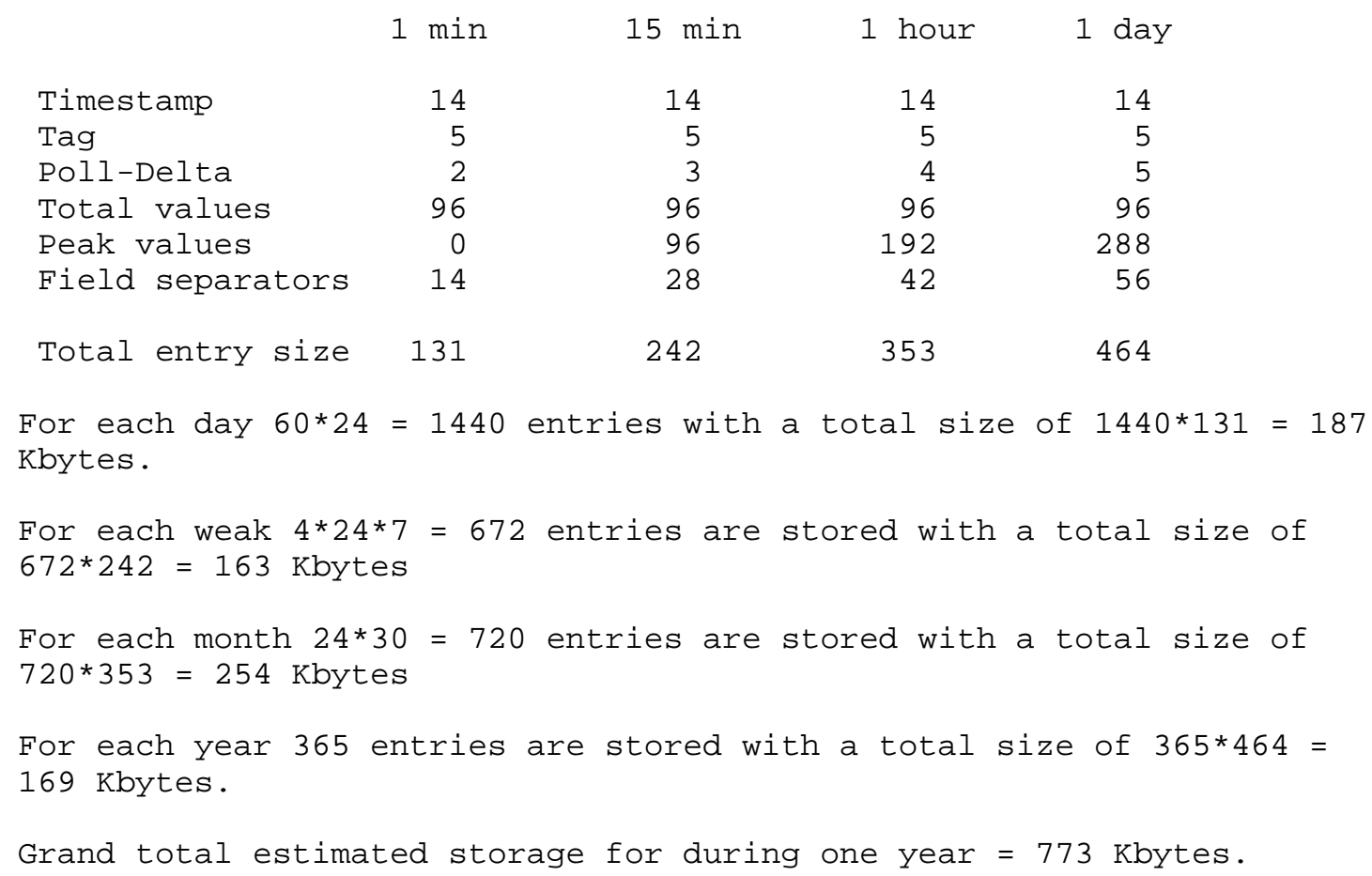




\section{Report Formats}

This section suggest some report formats and defines the metrics to be used in such reports.

\subsection{Report Types and Contents}

There is the longer term needs for monthly and yearly reports showing the long term tendencies in the network. There are the short term weekly reports giving indications on the medium term changes in the network behavior which could serve as input in the medium term engineering approach. Finally there is the daily reports giving instantaneous overviews needed in the daily operations of a network.

These reports should give information on:

Offered Load
Offered Load
Offered Load

Resource Utilization
Total traffic at external interfaces. Segmented by "Customer". Segmented protocol/application.

Link/Router.

7.2 Contents of the Reports

7.2.1 Offered Load by Link

Metric categories: input octets per external interface output octets per external interface input packets per external interface output packets per external interface

The intention is to visualize the overall trend of network traffic on each connected external interface. This could be done as a bar-chart giving the totals for each of the four metric categories. Based on the time period selected this could be done on a hourly, daily, monthly or yearly basis.

\subsubsection{Offered Load by Customer}

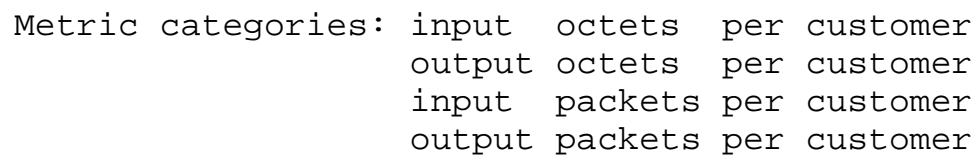

The recommendation is here to sort the offered load (in decreasing order) by customer. Plot the function $F(n)$, where $F(n)$ is percentage of total traffic offered to the top $n$ customers or the function $f(n)$ where $f$ is the percentage of traffic offered by the $n^{\prime}$ th ranked 
customers.

The definition of what should be meant by a customer has to be done locally at the site where the statistics are being gathered.

The cumulative could be useful as an overview of how the traffic is distributed among users since it enables to quickly pick off what fraction of of the traffic comes from what number of "users."

A method of displaying both average and peak-behaviors in the same bar-diagram is to compute both the average value over some period and the peak value during the same period. The average and peak values are then displayed in the same bar.

\subsubsection{Resource Utilization Reporting}

\subsubsection{Utilization as Maximum Peak Behavior}

The link utilization is used to capture information on network loading. The polling interval must be small enough to be significant with respect to variations in human activity since this is the activity that drives loading in network variation. On the other hand, there is no need to make it smaller than an interval over which excessive delay would notably impact productivity. For this reason 30 minutes is a good estimate the time at which people remain in one activity and over which prolonged high delay will affect their productivity. To track 30 minute variations, there is a need to sample twice as frequently, i.e., every 15 minutes. Using above recommended polling period of 10 minutes this will hence be sufficient to capture variations in utilizations.

A possible format for reporting utilizations seen as peak behaviors is to use a method of combining averages and peak measurements onto the same diagram. Compare for example peak-meters on audio-equipment. If for example a diagram contains the daily totals for some period, then the peaks would be the most busy hour during each day. If the diagram was totals on hourly basis then the peak would be the maximum 10 minutes period for each hour.

By combining the average and the maximum values for a certain timeperiod it will be possible to detect line utilization and bottlenecks due to temporary high loads.

\subsubsection{Utilization Visualized as a Frequency Distribution of Peaks}

Another way of visualizing line utilization is to put the 10 minutes samples in a histogram showing the relative frequency among the samples vs. the load. 
8. Considerations for Future Development

This memo is the first effort in formalizing a common basis for operational statistics. One major guideline in this work has been to keep the model simple to facilitate for vendors and Nocs to easily integrate this model in their operational tools.

There are, however, some ideas that could be progressed further to expand the scope and usability of the model.

\subsection{A Client/Server Based Statistical Exchange System}

A possible way of development could be the definition of a client/server based architecture for providing Internet access to operational statistics. Such an architecture envisions that each NoC should install a server who provides locally collected information in a variety of forms for clients.

Using a query language the client should be able to define the network object, the interface, the metrics and the time period to be provided. Using a TCP based protocol the server will transmit the requested data. Once these data is received by the client they could be processed and presented by a variety of tools needed. One possibility is to have an $\mathrm{X}$-Window based tool that displays defined diagrams from data, supporting such types of diagrams being feed into the $\mathrm{X}$-window tool directly from the statistical server. Another complementary method would be to generate Postscript output to be able to print the diagrams. In all cases there should be the possibility to store the retrieved data locally for later processing.

\subsection{Inclusion of Variables not in the Internet Standard MIB}

As has been pointed out above in the categorization of metrics there are metrics which certainly could have been recommended if being available in the Internet Standard MIB. To facilitate for such metrics to be part of the set of recommended metrics it will be necessary to specify a subtree in the Internet standard MIB containing variables judged necessary in the scope of performing operational statistics.

\subsection{Detailed Resource Utilization Statistics}

One area of interest not covered in the above description of metrics and presentation formats is to present statistics on detailed views of the traffic flows. Such views could include statistics on a per application basis and on a per protocol basis. Today such metrics are not part of the Internet Standard MIB. Tools like the NSF NNStat are being used to gather information of this kind. A possible way to 
achieve such data could be to define a NNStat MIB or to include such variables in the above suggested operational statistics MIB subtree. APPENDIX A

Some formulas for statistical aggregation

The following naming conventions are being used:

For poll values poll(n)_j

$\mathrm{n}=$ Polling or aggregation period

$j=$ Entry number

poll(900)_j is thus the 15 minute total value.

For peak values peak $(n, m) \_j$

$\mathrm{n}=$ Period over which the peak is calculated

$\mathrm{m}=$ The peak period length

$j=$ Entry number

peak $(3600,900) \ldots j$ is thus the maximum 15 minute period calculated over 1 hour.

Assume a polling over 24 hour period giving 1440 logged entries.

Without any aggregation we have

$\operatorname{poll}(60) \ldots 1$

......

$\operatorname{poll}(60) \_1439$

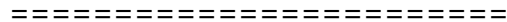

15 minute aggregation will give 96 entries of total values

poll (900)_1

$\cdots$

poll (900)_96

$$
j=(n+14)
$$




$$
\operatorname{poll}(900) \_k=\operatorname{SUM}_{j=n} \operatorname{poll}(60){ }_{j} j \quad \begin{aligned}
& n=1,16,31, \ldots 1425 \\
& k=1,2, \ldots .96
\end{aligned}
$$

There will also be 961 minute peak values.

$$
\operatorname{peak}(900,60) \_k=\underset{\substack{j=(n+14) \\
j=n}}{\operatorname{MAX} \operatorname{poll}(60) \_000 j \quad \begin{array}{l}
n=1,16,31, \ldots, 1425 \\
k=1,2, \ldots, 96
\end{array}}
$$

$==\mathrm{e}=\mathrm{=}=\mathrm{=}=\mathrm{=}=\mathrm{=}=\mathrm{=}=\mathrm{=}=\mathrm{=}=\mathrm{=}=\mathrm{=}$

Next aggregation step is from 15 minute to 1 hour.

This gives 24 totals

$$
\operatorname{poll}(3600) \_k=\operatorname{SUM} \begin{aligned}
& j=(n+3) \\
& \begin{array}{l}
j=n \\
j=11(900)
\end{array} j
\end{aligned} \quad \begin{aligned}
& n=1,5,9, \ldots \ldots, 93 \\
& k=1,2, \ldots, 24
\end{aligned}
$$

and 241 minute peaks calculated over each hour.

$$
\text { peak }(3600,60) \_k=\underset{j=n}{\operatorname{MAX}} \operatorname{peak}(900,60) \_j \quad \begin{aligned}
& n=1,5,9, \ldots \ldots, 93 \\
& k=1,2, \ldots .24
\end{aligned}
$$

and finally 2415 minute peaks calculated over each hour.

$$
\text { peak }(3600,900)=\underset{\substack{j=(n+3) \\ j=n}}{\operatorname{MAX} \operatorname{poll}(900) \_j \quad n=1,5,9, \ldots ., 93}
$$

Next aggregation step is from 1 hour to 24 hour

For each day with 1440 entries as above this will give

$$
j=(n+23)
$$




$$
\begin{aligned}
& \operatorname{poll}(86400) \_k=\operatorname{SUM}_{j=n} \operatorname{poll}(3600){ }_{j}^{j} \quad \begin{array}{l}
n=1,25,51, \ldots \ldots \ldots \\
k=1,2 \ldots \ldots
\end{array} \\
& \text { peak }(86400,60) \_k \quad \begin{array}{l}
j=(n+23) \\
\operatorname{MAX} \operatorname{peak}(3600,60) \_j
\end{array} \quad \begin{array}{l}
n=1,25,51, \ldots \ldots \\
k=1,2 \ldots \ldots
\end{array}
\end{aligned}
$$

which gives the busiest 1 minute period over 24 hours.

$$
\text { peak }(86400,900) \_k=\begin{aligned}
& j=(n+23) \\
& \operatorname{MAX} \operatorname{peak}(3600,900) \_j
\end{aligned} \quad \begin{aligned}
& n=1,25,51, \ldots \ldots \\
& k=1,2, \ldots
\end{aligned}
$$

which gives the busiest 15 minute period over 24 hours.

$$
\operatorname{peak}(86400,3600) \_k=\begin{aligned}
& j=(n+23) \\
& \operatorname{MAX} \operatorname{poll}(3600) \_j \quad \begin{array}{l}
n=1,25,51, \ldots . \\
k=1,2, \ldots \ldots
\end{array}
\end{aligned}
$$

which gives the busiest 1 hour period over 24 hours.

There will probably be a difference between the three peak values in the final 24 hour aggregation. Smaller peak period will give higher values than longer, $i . e .$, if adjusted to be numerically comparable.

$\operatorname{poll}(86400) / 3600<\operatorname{peak}(86400,3600)<\operatorname{peak}(86400,900) * 4$

$<\operatorname{peak}(86400,60) * 60$ 


\section{APPENDIX B}

An example

Assuming below data storage:

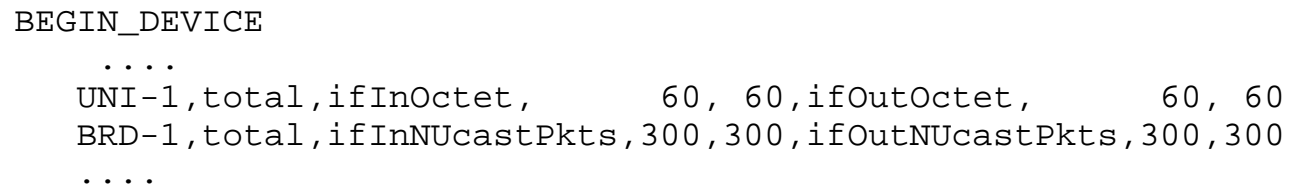




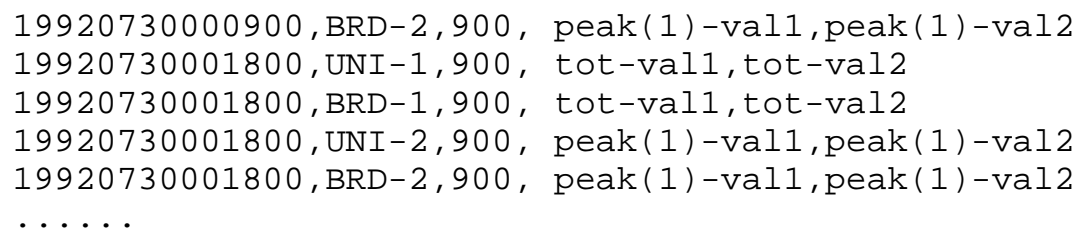

Finally aggregation step to 1 day generates:

UNI-1, total, ifInoctet, 60, 86400 , ifoutoctet, 60,86400 
BRD-1, total, ifInNUcastPkts, 300,86400, ifOutNUcastPkts, 300, 86400
UNI-2, peak, ifInOctet, 60,86400, ifOutOctet, 60, 86400
BRD-2, peak, ifInNUcastPkts, 300,900, ifOutNUcastPkts, 300,900
UNI-3, peak, ifInOctet, 900,86400, ifOutOctet, 900,86400
BRD-3, peak, ifInNUcastPkts, 900,86400, ifOutNUcastPkts, 900,86400
UNI-4, peak, ifInOctet, 3600,86400, ifOutOctet, 3600,86400
BRD-4, peak, ifInNUcastPkts, 3600,86400, ifOutNUcastPkts, 3600,86400

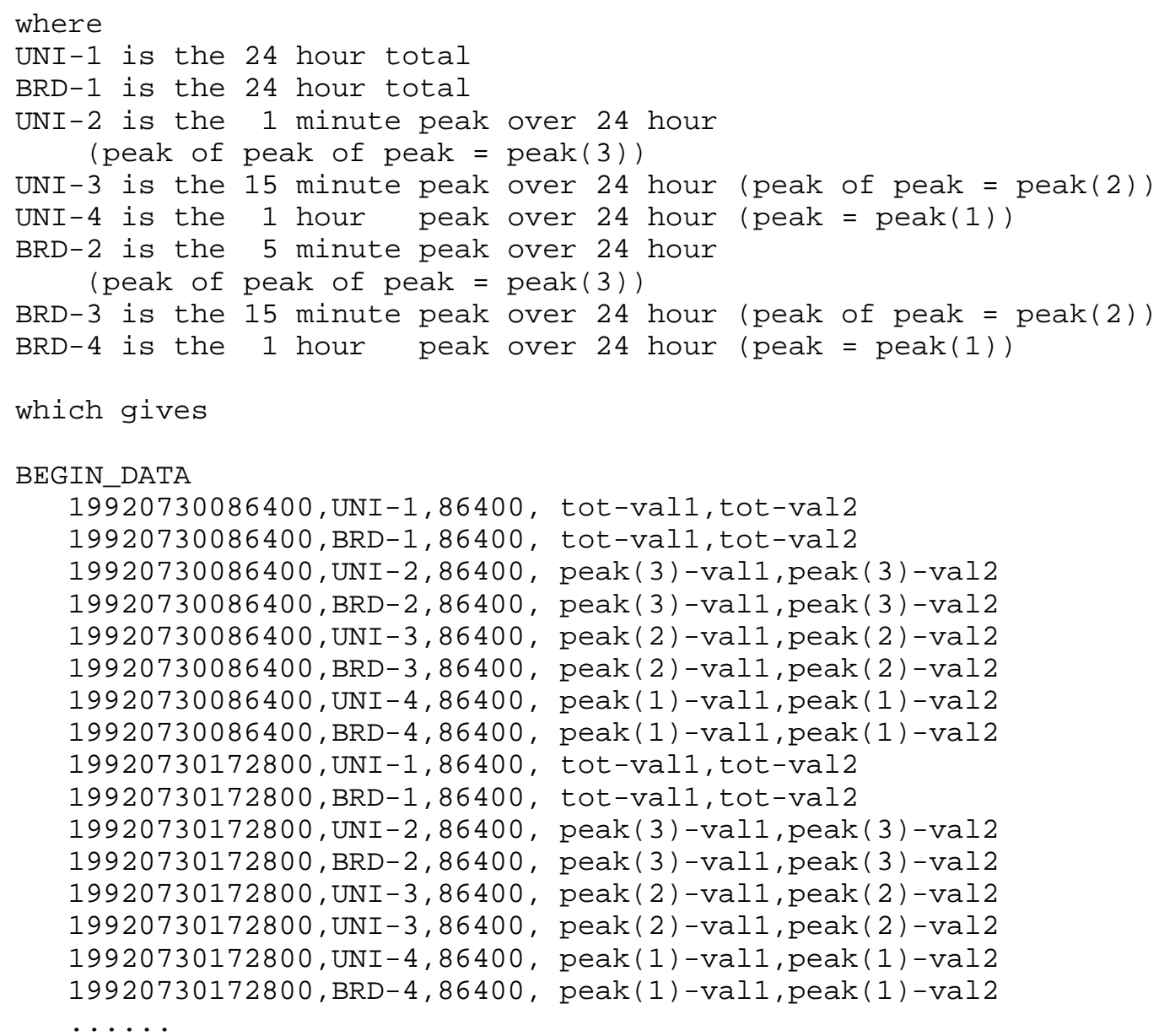




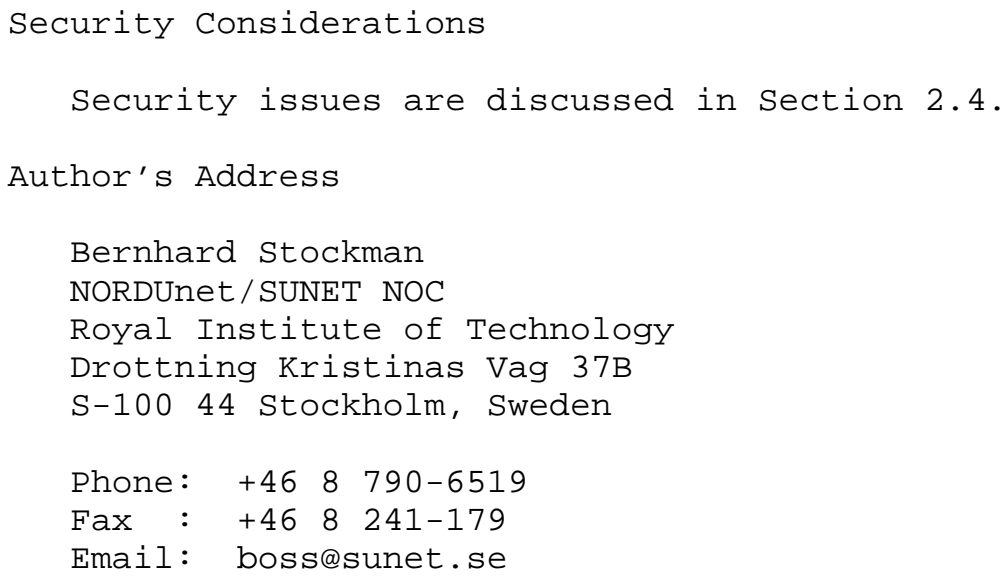

Email: boss@sunet.se 